\title{
078 Nematode faunal analyses to assess food web enrichment and connectance
}

Howard FERris ${ }^{1, *}$, Tom BONGERS ${ }^{2}$ and

Ron DeGoedE ${ }^{3}$

${ }^{1}$ Department of Nematology, University of California, Davis, CA 95616, USA

${ }^{2}$ Laboratory of Nematology, Wageningen University, P.O. Box 8123, 6700 ES Wageningen, The Netherlands

${ }^{3}$ Sub-department of Soil Quality, Wageningen University, P.O. Box 8055, 6700 EC Wageningen, The Netherlands

*hferris@ucdavis.edu.

The cp classification recognises that nematode taxa with anatomical and physiological commonalities are probably similarly adapted to specific environmental conditions. Functional guild analysis, which integrates cpscaling with food sources, reveals that some guilds respond opportunistically to enrichment while others represent the presence of higher trophic connectance in the food web. This dichotomy was portrayed graphically in $\mathrm{cp}$ triangles as the proportional representation of enrichment opportunists (cp 1), general opportunists (cp 2) and taxa indicating higher connectance (cp 3-5). Confounded in the calculation of cp triangles is interdependence of the axes; proportionality of the three groupings to the whole nema- tode fauna requires that increase in food web structure (cp 3-5 taxa) concomitantly decreases the enrichment indicator (cp 1 taxa). The categorical separation of nematode taxa into five cp classes does not imply unit increments in $\mathrm{r}$ or $\mathrm{K}$ characteristics. We use body size and growth rates to weight the importance of enrichment indicators and estimates of corresponding food web connectance to weight the importance of structure indicators. We consider cp 2 taxa basal to both trajectories and calculate position along the enrichment and structure axes of a faunal diagram independently as the weighted ratios of the indicator and basal taxa. 